Research Article

\title{
Some Weighted Estimates for Multilinear Fourier Multiplier Operators
}

\author{
Zengyan Si \\ School of Mathematics and Information Science, Henan Polytechnic University, Jiaozuo 454000, China \\ Correspondence should be addressed to Zengyan Si; zengyan@hpu.edu.cn
}

Received 3 August 2013; Revised 2 October 2013; Accepted 20 October 2013

Academic Editor: Mieczysław Mastyło

Copyright (C) 2013 Zengyan Si. This is an open access article distributed under the Creative Commons Attribution License, which permits unrestricted use, distribution, and reproduction in any medium, provided the original work is properly cited.

We first provide a weighted Fourier multiplier theorem for multilinear operators which extends Theorem 1.2 in Fujita and Tomita (2012) by using $L^{r}$-based Sobolev spaces $(1<r \leq 2)$. Then, by using a different method, we obtain a result parallel to Theorem 6.2 which is an improvement of Theorem 1.2 under assumption (i) in Fujita and Tomita (2012).

\section{Introduction}

During the last several years, considerable attention has been paid to the study of multilinear Fourier multiplier operators. Let $\delta\left(\mathbb{R}^{d}\right)$ be the Schwartz space of all rapidly decreasing smooth functions on $\mathbb{R}^{d}$, for some $d \in \mathbb{Z}^{+}$. The multilinear Fourier multiplier operator $T_{\sigma}$ associated with a symbol $\sigma$ is defined by

$$
\begin{aligned}
T_{\sigma}\left(f_{1}, \ldots, f_{m}\right)(x) \\
=\int_{\mathbb{R}^{m n}} e^{2 \pi i x \cdot\left(\xi_{1}+\cdots+\xi_{m}\right)} \sigma\left(\xi_{1}, \ldots, \xi_{m}\right) \\
\quad \times \widehat{f}_{1}\left(\xi_{1}\right) \cdots \widehat{f}_{m}\left(\xi_{m}\right) d \xi_{1} \cdots d \xi_{m}
\end{aligned}
$$

for $f_{i} \in \mathcal{S}\left(\mathbb{R}^{n}\right), i=1, \ldots, m$

Coifman and Meyer [1] proved that if $\sigma$ is a bounded function on $\mathbb{R}^{m n} \backslash\{0\}$ that satisfies

$$
\left|\partial_{\xi_{1}}^{\alpha_{1}} \cdots \partial_{\xi_{m}}^{\alpha_{m}} \sigma\left(\xi_{1}, \ldots, \xi_{m}\right)\right| \leq C_{\alpha}\left(\left|\xi_{1}\right|+\cdots+\left|\xi_{m}\right|\right)^{-\left(\left|\alpha_{1}\right|+\cdots+\left|\alpha_{m}\right|\right)}
$$

away from the origin for all sufficiently large multi-indices $\alpha_{j}$, then $T_{\sigma}$ is bounded from the product $L^{p_{1}}\left(\mathbb{R}^{n}\right) \times \cdots \times$ $L^{p_{m}}\left(\mathbb{R}^{n}\right)$ to $L^{p}\left(\mathbb{R}^{n}\right)$ for all $1<p_{1}, \ldots, p_{m}, p<\infty$ satisfying $1 / p_{1}+\cdots+1 / p_{m}=1 / p$. The multiplier theorem of Coifman and Meyer was extended to indices $p<1$ (and larger than $1 / m)$ by Grafakos and Torres [2] and Kenig and Stein [3] (when $m=2$ ). Exploiting the idea of the proof of the Hörmander multiplier theorem in [4], Tomita [5] gave a Hörmander type theorem for multilinear Fourier multipliers with more weaker smoothness condition assumed on $\sigma$ than (2). Grafakos and Si [6] gave similar results for $p \leq 1$ by using $L^{r}$-based Sobolev spaces $(1<r \leq 2)$. Grafakos et al. [7] proved the $L^{2}$-boundedness of $T_{\sigma}$ with multipliers of limited smoothness.

In order to state other known results, we first introduce some notations. The Laplacian on $\mathbb{R}^{d}$ is $\Delta g=\sum_{j=1}^{d} \partial^{2} g / \partial x_{j}^{2}$, that is, the sum of the second partials of $g$ in every variable. We define the operator $(I-\Delta)^{\gamma / 2}(g)=\mathscr{F}^{-1}\left(w_{\gamma} \mathscr{F}(g)\right)$, where $w_{\gamma}(\xi)=\left(1+4 \pi^{2}|\xi|^{2}\right)^{\gamma / 2}$ for $\gamma>0$. Let $L_{\gamma}^{r}\left(\mathbb{R}^{d}\right)$ be the $L^{r}$-based Sobolev space with norm

$$
\|f\|_{L_{\gamma}^{r}}=\left\|(I-\Delta)^{\gamma / 2} f\right\|_{L^{r}\left(\mathbb{R}^{d}\right)},
$$

where $1 \leq r<\infty$.

Let $\vec{s}=\left(s_{1}, \ldots, s_{m}\right)$ and let the product type Sobolev space $W^{s_{1}, \ldots, s_{m}}\left(\mathbb{R}^{m n}\right)$ consist of all functions $F$ such that the following norm of $F$ is finite:

$$
\begin{aligned}
& \|F\|_{W^{s_{1}, \ldots, s_{m}\left(\mathbb{R}^{m n}\right)}} \\
& \quad=\left(\int_{\mathbb{R}^{m n}}\left\langle\xi_{1}\right\rangle^{2 s_{1}} \cdots\left\langle\xi_{m}\right\rangle^{2 s_{m}}|\widehat{F}(\xi)|^{2} d \xi\right)^{1 / 2},
\end{aligned}
$$

where $\xi=\left(\xi_{1}, \ldots, \xi_{m}\right)$ and $\left\langle\xi_{k}\right\rangle=\left(1+\left|\xi_{k}\right|^{2}\right)^{1 / 2}$. 
Let $\psi \in \mathcal{S}\left(\mathbb{R}^{m n}\right)$ be such that supp $\psi \subset\left\{\xi \in \mathbb{R}^{m n}: 1 / 2 \leq\right.$ $|\xi| \leq 2\}$ and $\sum_{j \in \mathbb{Z}} \psi\left(2^{-j} \xi\right)=1$ for $\xi \neq 0$.

Let $\mathcal{S}_{1}\left(\mathbb{R}^{d}\right)$ be the set of all Schwartz functions $\Psi$ on $\mathbb{R}^{d}$, whose Fourier transform is supported in an annulus of the form $\left\{\xi: c_{1}<|\xi|<c_{2}\right\}$, is nonvanishing in a smaller annulus $\left\{\xi: c_{1}^{\prime} \leq|\xi| \leq c_{2}^{\prime}\right\}$ (for some choice of constants $0<c_{1}<c_{1}^{\prime}<$ $\left.c_{2}^{\prime}<c_{2}<\infty\right)$, and satisfies

$$
\sum_{j \in \mathbb{Z}} \widehat{\Psi}\left(2^{-j} \xi\right)=\text { constant, } \quad \xi \in \mathbb{R}^{d} \backslash\{0\} .
$$

The weighted estimate for $T_{\sigma}$ is also an interesting topic in harmonic analysis. And it has attracted many authors in this area. Recently, Fujita and Tomita [8] established some weighted estimates of $T_{\sigma}$ under the Hörmander condition and classical $A_{p}$ weights. For other works about the weighted estimates for $T_{\sigma}$, see $[9,10]$ and the references therein.

Theorem A (see [8]). Let $1<p_{1}, p_{2}, \ldots, p_{N}<\infty, 1 / p_{1}+\cdots+$ $1 / p_{N}=1 / p$, and $N n / 2<s \leq N n$. Assume

(i) $\min p_{1}, \ldots, p_{N}>N n / s$ and $\omega \in A_{\min p_{1} s / N n, \ldots, p_{N} s / N n}$;

(ii) $\min p_{1}, \ldots, p_{N}>(N n / s)^{\prime}$ and $1<p<\infty, \omega^{1-p^{\prime}} \epsilon$ $A_{p^{\prime} s /(\mathrm{Nn})}$.

If $\sigma \in L^{\infty}$ satisfies $\sup _{k \in Z}\left\|\sigma\left(2^{k} \cdot\right) \psi\right\|_{L_{s}^{2}}<\infty$, then $T_{\sigma}$ is bounded from $L^{p_{1}}(\omega) \times \cdots \times L^{p_{m}}(\omega)$ to $L^{p}(\omega)$.

An improvement of Theorem 1.2 is stated as follows.

Theorem B (see [8]). Let $1<p_{1}, p_{2}, \ldots, p_{N}<\infty, 1 / p_{1}+$ $\cdots+1 / p_{N}=1 / p$, and $n / 2<s_{j} \leq n, j=1, \ldots, N$. Assume $p_{j}>n / s_{j}$ and $\omega_{j} \in A_{p_{j} s_{j} / n}$ for $1 \leq j \leq N$. If $\sigma \in L^{\infty}$ satisfies $\sup _{k \in Z}\left\|\sigma\left(2^{k} \cdot\right) \psi\right\|_{W^{\left(s_{1}, \cdots, s_{N}\right)}\left(R^{N n}\right)}<\infty$, then $T_{\sigma}$ is bounded from $L^{p_{1}}\left(\omega_{1}\right) \times \cdots \times L^{p_{m}}\left(\omega_{N}\right)$ to $L^{p}(\omega)$, where $\omega=\omega_{1}^{p / p_{1}} \cdots \omega_{N}^{p / p_{N}}$.

The first purpose of this paper is to improve Theorem A by using $L^{r}$-based Sobolev spaces $(1<r \leq 2)$. The second purpose is to give a new proof of Theorem $\mathrm{B}$. The following are the main results.

Theorem 1. For some $1<r \leq 2$, suppose that $\sigma \in L^{\infty}\left(\mathbb{R}^{m n}\right)$ and $\Psi \in \mathcal{S}_{1}\left(\mathbb{R}^{m n}\right)$ satisfy, for some $m n / r<\gamma \leq m n$,

$$
\sup _{k \in \mathbb{Z}} \| \sigma\left(2^{k} \cdot \widehat{\Psi} \|_{L_{\gamma}^{r}\left(\mathbb{R}^{m n}\right)}=K<\infty .\right.
$$

If $p_{1}, \ldots, p_{m}, \gamma$, and the weights $\omega$ satisfy one of the following two conditions:

(i) $\min \left\{p_{1}, \ldots, p_{m}\right\}>m n / \gamma$ and $\omega \in$ $A_{\min \left\{p_{1} \gamma /(m n), \ldots, p_{m} \gamma /(m n)\right\}}$,

(ii) $\min \left\{p_{1}, \ldots, p_{m}\right\}>(m n / \gamma)^{\prime}, 1<p<\infty$, and $\omega^{1-p^{\prime}} \epsilon$ $A_{p^{\prime} \gamma /(m n)}$,

then there is a number $\delta=\delta(m n, \gamma, r)$ satisfying $0<\delta \leq$ $r-1$, such that the m-linear operator $T_{\sigma}$, associated with the multiplier $\sigma$, is bounded from $L^{p_{1}}(\omega) \times \cdots \times L^{p_{m}}(\omega)$ to $L^{p}(\omega)$, whenever $r-\delta<p_{j}<\infty$ for all $j=1, \ldots, m$, and $p$ is given by $1 / p=1 / p_{1}+\cdots+1 / p_{m}$.
Theorem 2. Let $1<p_{1}, \ldots, p_{m}<2$ and let $s_{1}>n / p_{1}, \ldots$, $s_{m}>n / p_{m}$ and $s_{1}+\cdots+s_{m}<n / p_{1}+\cdots+n / p_{m}+1$. If $\sigma \epsilon$ $L^{\infty}\left(\mathbb{R}^{m n}\right)$ satisfies $\sup _{k \in \mathbb{Z}}\left\|\sigma\left(2^{k} \cdot\right) \psi\right\|_{W^{s_{1}, \ldots, s_{m}\left(\mathbb{R}^{m n}\right)}}<\infty$, then $T_{\sigma}$ is bounded from $L^{q_{1}}\left(w_{1}^{q_{1}}\right) \times \cdots \times L^{q_{m}}\left(w_{m}^{q_{m}}\right)$ to $L^{q}\left(w^{q}\right)$, whenever $1<q_{1}, \ldots, q_{m}<\infty, 1 / q_{1}+\cdots+1 / q_{m}=1 / q$, and $\left(w_{1}^{q_{1}}, \ldots, w_{m}^{q_{m}}\right) \in\left(A_{q_{1}}, \ldots, A_{q_{m}}\right)$ with $w=w_{1} \cdots w_{m}$.

\section{The Proof of Theorem 1}

In this section we discuss the proof of Theorem 1 . We begin with some definitions for maximal operators. Throughout the paper, $M$ denotes the Hardy-Littlewood maximal operator defined by

$$
M(f)(x)=\sup _{x \in Q} \frac{1}{|Q|} \int_{Q}|f(y)| d y,
$$

where $Q$ moves over all cubes containing $x$. For $\delta>0, M_{\delta}$ is the maximal function defined by

$$
M_{\delta} f(x)=M\left(|f|^{\delta}\right)^{1 / \delta}(x)=\left(\sup _{Q \ni x} \frac{1}{|Q|} \int_{Q}|f(y)|^{\delta} d y\right)^{1 / \delta} .
$$

In addition, $M^{\sharp}$ is the sharp maximal function of Fefferman and Stein:

$$
\begin{aligned}
M^{\sharp} f(x) & =\sup _{\mathrm{Q} \ni x} \inf _{c} \frac{1}{|Q|} \int_{\mathrm{Q}}|f(y)-c| d y \\
& \approx \sup _{\mathrm{Q} \ni x} \frac{1}{|Q|} \int_{\mathrm{Q}}\left|f(y)-f_{\mathrm{Q}}\right| d y,
\end{aligned}
$$

where $f_{\mathrm{Q}}$ denotes the average of $f$ over $Q$ and a variant of $M^{\sharp}$ is given by

$$
M_{\delta}^{\sharp} f(x)=M^{\sharp}\left(|f|^{\delta}\right)^{1 / \delta}(x) .
$$

We prepare some lemmas which will be used later.

Lemma 3 (see [11]). Let $1<p<\infty$ and $\omega \in A_{p}$. Then (1) $\omega^{1-p^{\prime}} \in A_{p^{\prime}}$; (2) there exists $q<p$ such that $\omega \in A_{q}$.

Lemma 4 (see [12]). Let $1<p, q<\infty$, and $\omega \in A_{p}$. Then there exist positive finite constants $C(p, q)$ such that

$$
\left\|\left\{\sum_{k \in \mathbb{Z}}\left|M\left(f_{k}\right)\right|^{q}\right\}^{1 / q}\right\|_{L^{p}(\omega)} \leq C(p, q)\left\|\left\{\sum_{k \in \mathbb{Z}}\left|f_{k}\right|^{q}\right\}^{1 / q}\right\|_{L^{p}(\omega)}
$$

for all sequences $\left\{f_{k}\right\}_{k \in \mathbb{Z}}$ of locally integrable functions on $\mathbb{R}^{n}$.

Lemma 5. Let $\Delta_{k}$ be the Littlewood-Paley operator given by $\Delta_{k}(g)^{-}(\xi)=\widehat{g}(\xi) \widehat{\Psi}\left(2^{-k} \xi\right), k \in \mathbb{Z}$, where $\Psi$ is a Schwartz function whose Fourier transform is supported in the annulus $\left\{\xi: 2^{-b}<|\xi|<2^{b}\right\}$, for some $b \in \mathbb{Z}^{+}$, and satisfies $\sum_{k \in \mathbb{Z}} \widehat{\Psi}\left(2^{-k} \xi\right)=c_{0}$, for some constant $c_{0}$. Let $0<p<\infty$ 
and $\omega \in A_{\infty}$. Then there is a constant $c=c\left(n, p, c_{0}, \Psi\right)$, such that for $L^{p}(\omega)$ functions $f$ one has

$$
\|f\|_{L^{p}(\omega)} \leq c\left\|\left(\sum_{k \in \mathbb{Z}}\left|\Delta_{k}(f)\right|^{2}\right)^{1 / 2}\right\|_{L^{p}(\omega)} .
$$

Proof. The proof follows from similar steps in Lemma 4 of [6] and combines the method used in Remark 2.6 of [8]. Let $\Phi$ be a Schwartz function with integral one. Then,

$$
|f(x)|=\lim _{t \rightarrow 0}\left|\Phi_{t} * f(x)\right| \leq \sup _{t>0}\left|\Phi_{t} * f(x)\right| .
$$

If $\omega \in A_{\infty}$, the weighted Hardy space $H^{p}(\omega)$ coincides with the weighted Triebel-Lizorkin space $\dot{F}_{0}^{p, 2}$ for $0<p<\infty$. Hence, if $\omega \in A_{\infty}$, we have

$$
\begin{aligned}
\|f\|_{L^{p}(\omega)} & \leq\left\|\sup _{t>0}\left|\Phi_{t} * f(x)\right|\right\|_{L^{p}(\omega)} \\
& =c\|f\|_{H^{p}(\omega)} \approx\|f\|_{\dot{F}_{0}^{p, 2}(\omega)} \\
& \leq c\left\|\left(\sum_{k \in \mathbb{Z}}\left|\Delta_{k}(f)\right|^{2}\right)^{1 / 2}\right\|_{L^{p}(\omega)} .
\end{aligned}
$$

The proof is complete.

Now we give the proof of Theorem 1.

Proof. Since the proof follows from similar steps in Theorem 1 in [6], we just give the different parts. For each $j=1, \ldots, m$, we let $R_{j}$ be the set of points $\left(\xi_{1}, \ldots, \xi_{m}\right)$ in $\left(\mathbb{R}^{n}\right)^{m}$ such that $\left|\xi_{j}\right|=\max \left\{\left|\xi_{1}\right|, \ldots,\left|\xi_{m}\right|\right\}$ and we introduce nonnegative smooth functions $\phi_{j}$ on $[0, \infty)^{m-1}$ that are supported in $[0,11 / 10]^{m-1}$ such that

$$
1=\sum_{j=1}^{m} \phi_{j}\left(\frac{\left|\xi_{1}\right|}{\left|\xi_{j}\right|}, \ldots, \frac{\widehat{\left|\xi_{j}\right|}}{\left|\xi_{j}\right|}, \ldots, \frac{\left|\xi_{m}\right|}{\left|\xi_{j}\right|}\right)
$$

for all $\left(\xi_{1}, \ldots, \xi_{m}\right) \neq 0$, with the understanding that the variable with the hat is missing. These functions introduce a partition of unity of $\left(\mathbb{R}^{n}\right)^{m} \backslash\{0\}$ subordinate to a conical neighborhood of the region $R_{j}$.

Each region $R_{j}$ can be written as the union of sets:

$$
R_{j, k}=\left\{\left(\xi_{1}, \ldots, \xi_{m}\right) \in R_{j}:\left|\xi_{k}\right| \geq\left|\xi_{s}\right| \forall s \neq j\right\}
$$

with $k=1, \ldots, m$. We need to work with a finer partition of unity, subordinate to each $R_{j, k}$. To achieve this, for each $j$, we introduce smooth functions $\phi_{j, k}$ on $[0, \infty)^{m-2}$ supported in $[0,11 / 10]^{m-2}$ such that

$$
1=\sum_{\substack{k=1 \\ k \neq j}}^{m} \phi_{j, k}\left(\frac{\left|\xi_{1}\right|}{\left|\xi_{k}\right|}, \ldots, \frac{\widehat{\left|\xi_{k}\right|}}{\left|\xi_{k}\right|}, \ldots, \frac{\widehat{\left|\xi_{j}\right|}}{\left|\xi_{k}\right|}, \ldots, \frac{\left|\xi_{m}\right|}{\left|\xi_{k}\right|}\right)
$$

for all $\left(\xi_{1}, \ldots, \xi_{m}\right)$ in the support of $\phi_{j}$ with $\xi_{k} \neq 0$.
We now have obtained the following partition of unity of $\left(\mathbb{R}^{n}\right)^{m} \backslash\{0\}:$

$$
1=\sum_{j=1}^{m} \sum_{\substack{k=1 \\ k \neq j}}^{m} \phi_{j}(\cdots) \phi_{j, k}(\cdots)
$$

where the dots indicate the variables of each function.

We now introduce a nonnegative smooth bump $\psi$ supported in the interval $\left[(10 m)^{-1}, 2\right]$ and equal to 1 in the interval $\left[(5 m)^{-1}, 12 / 10\right]$ and we decompose $\sigma$ into a finite number of multipliers:

$$
\sigma=\sum_{j=1}^{m} \sum_{\substack{k=1 \\ k \neq j}}^{m}\left[\sigma \Phi_{j, k}+\sigma \Psi_{j, k}\right]
$$

where

$$
\begin{gathered}
\Phi_{j, k}\left(\xi_{1}, \ldots, \xi_{m}\right)=\phi_{j}(\cdots) \phi_{j, k}(\cdots)\left(1-\psi\left(\frac{\left|\xi_{k}\right|}{\left|\xi_{j}\right|}\right)\right), \\
\Psi_{j, k}\left(\xi_{1}, \ldots, \xi_{m}\right)=\phi_{j}(\cdots) \phi_{j, k}(\cdots) \psi\left(\frac{\left|\xi_{k}\right|}{\left|\xi_{j}\right|}\right) .
\end{gathered}
$$

We will prove the required assertion for each piece of this decomposition, that is, for the multipliers $\sigma \Phi_{j, k}$ and $\sigma \Psi_{j, k}$ for each pair $(j, k)$ in the previous sum. In view of the symmetry of the decomposition, it suffices to consider the case of a fixed pair $(j, k)$ in the previous sum. To simplify notation, we fix the pair $(m, m-1)$; thus, for the rest of the proof we fix $j=m$ and $k=m-1$ and we prove boundedness for the $m$-linear operators whose symbols are $\sigma_{1}=\sigma \Phi_{m, m-1}$ and $\sigma_{2}=\sigma \Psi_{m, m-1}$. These correspond to the $m$-linear operators $T_{\sigma_{1}}$ and $T_{\sigma_{2}}$, respectively.

We first prove Theorem 1 under assumption (i). Since $1 \leq m n / \gamma<\min \left\{r, p_{1}, \ldots, p_{m}\right\}$, we can take $\rho$ such that $1 \leq$ $m n / \gamma<\rho<\min \left\{r, p_{1}, \ldots, p_{m}\right\}$ and $\omega \in A_{\min \left\{p_{1} / \rho, \ldots, p_{m} / \rho\right\}}$. We first consider $T_{\sigma_{1}}\left(f_{1}, \ldots, f_{m}\right)$, where $f_{j}$ are fixed Schwartz functions. We fix a Schwartz radial function $\eta$ whose Fourier transform is supported in the annulus $1-(1 / 25) \leq|\xi| \leq 2$ and satisfies

$$
\sum_{j \in \mathbf{Z}} \widehat{\eta}\left(2^{-j} \xi\right)=1, \quad \xi \in \mathbb{R}^{n} \backslash\{0\} .
$$

Associated with $\eta$ we define the Littlewood-Paley operator $\Delta_{j}(f)=f * \eta_{2^{-j}}$, where $\eta_{t}(x)=t^{-n} \eta\left(t^{-1} x\right)$ for $t>0$. We also define an operator $S_{j}$ by setting

$$
S_{j}(g)=g * \zeta_{2^{-j}},
$$

where $\zeta$ is a smooth function whose Fourier transform is equal to 1 on the ball $|z|<3 / 5 m$ and vanishes outside 
the double of this ball. As in [6, page 143], by using Lemma 5 we get

$$
\begin{aligned}
& \left\|T_{\sigma_{1}}\left(f_{1}, \ldots, f_{m}\right)\right\|_{L^{p}(\omega)} \\
& \leq C\left\|\left[\sum_{j}\left|T_{\sigma_{1}}\left(S_{j}\left(f_{1}\right), \ldots, S_{j}\left(f_{m-1}\right), \Delta_{j}\left(f_{m}\right)\right)\right|^{2}\right]^{1 / 2}\right\|_{L^{p}(\omega)} .
\end{aligned}
$$
145]):

We will use the following estimate for $T_{\sigma_{1}}$ (see $[6$, page

$$
\begin{aligned}
& \left|T_{\sigma_{1}}\left(S_{j}\left(f_{1}\right), \ldots, S_{j}\left(f_{m-1}\right), \Delta_{j}\left(f_{m}\right)\right)\right| \\
& \quad \leq C K \prod_{i=1}^{m-1}\left(M\left(M\left(f_{i}\right)^{\rho}\right)\right)^{1 / \rho}\left(M\left(\left|\Delta_{j}\left(f_{m}\right)\right|^{\rho}\right)\right)^{1 / \rho} .
\end{aligned}
$$

We now square the previous expression, we sum over $j \epsilon$ $\mathbb{Z}$, and we take square roots. Since $r-\delta=\rho$, the hypothesis $p_{j}>r-\delta$ implies $p_{j}>\rho$, and thus each term $\left(M\left(M\left(f_{i}\right)^{\rho}\right)\right)^{1 / \rho}$ is bounded on $L^{p_{j}}(\omega)$. We obtain

$$
\begin{aligned}
& \left\|T_{\sigma_{1}}\left(f_{1}, \ldots, f_{m-1}, f_{m}\right)\right\|_{L^{p}(\omega)} \\
& \leq C K \|\left\{\sum_{j} \mid T_{\sigma_{1}}\left(S_{j}\left(f_{1}\right), \ldots, S_{j}\left(f_{m-1}\right),\right.\right. \\
& \left.\left.\Delta_{j}\left(f_{m}\right)\right)\left.\right|^{2}\right\}^{1 / 2} \|_{L^{p}(\omega)} \\
& \leq C^{\prime} K\left\|\left\{\sum_{j} M\left(\left|\Delta_{j}\left(f_{m}\right)\right|^{\rho}\right)^{2 / \rho}\right\}^{1 / 2}\right\|_{L^{p_{m}}(\omega)} \\
& \times \prod_{i=1}^{m-1}\left\|\left(M\left(M\left(f_{i}\right)^{\rho}\right)\right)^{1 / \rho}\right\|_{L^{p_{i}}(\omega)} \\
& \leq C^{\prime \prime} K\left\|\left\{\sum_{j} M\left(\left|\Delta_{j}\left(f_{m}\right)\right|^{\rho}\right)^{2 / \rho}\right\}^{\rho / 2}\right\|_{L^{p_{m} / \rho}(\omega)}^{1 / \rho} \prod_{i=1}^{m-1}\left\|f_{i}\right\|_{L^{p_{i}}(\omega)} \\
& \leq C^{\prime \prime} K \prod_{i=1}^{m}\left\|f_{i}\right\|_{L^{p_{i}}(\omega)}
\end{aligned}
$$

where the last step holds due to Lemma 4 with $q=2 / \rho$ and the weighted Littlewood-Paley theorem.

Next we deal with $\sigma_{2}$. Following [6, page 146], we write

$$
\begin{aligned}
T_{\sigma_{2}} & \left(f_{1}, \ldots, f_{m-1}, f_{m}\right) \\
& =\sum_{j \in \mathbb{Z}} T_{\sigma_{2}}\left(S_{j}^{\prime}\left(f_{1}\right), \ldots, S_{j}^{\prime}\left(f_{m-2}\right), \Delta_{j}^{\prime}\left(f_{m-1}\right), \Delta_{j}\left(f_{m}\right)\right),
\end{aligned}
$$

$$
\begin{aligned}
\left|T_{\sigma_{2}}\left(S_{j}^{\prime}\left(f_{1}\right), \ldots, S_{j}^{\prime}\left(f_{m-2}\right), \Delta_{j}^{\prime}\left(f_{m-1}\right), \Delta_{j}\left(f_{m}\right)\right)\right| \\
\leq C K \prod_{i=1}^{m-2}\left(M\left(M\left(f_{i}\right)^{\rho}\right)\right)^{1 / \rho}\left(M\left(\left|\Delta_{j}^{\prime}\left(f_{m-1}\right)\right|^{\rho}\right)\right)^{1 / \rho} \\
\quad \times\left(M\left(\left|\Delta_{j}\left(f_{m}\right)\right|^{\rho}\right)\right)^{1 / \rho},
\end{aligned}
$$

for some other Littlewood-Paley operator $\Delta_{j}^{\prime}$ which is given on the Fourier transform by multiplication with a bump $\widehat{\Theta}\left(2^{-j} \xi\right)$, where $\widehat{\Theta}$ is equal to one on the annulus $\left\{\xi \in \mathbb{R}^{n}\right.$ : $(24 / 25) \cdot(1 / 10 m) \leq|\xi| \leq 4\}$ and vanishes on a larger annulus. Also, $S_{j}^{\prime}$ is given by convolution with $\zeta_{2^{-j}}^{\prime}$, where $\zeta^{\prime}$ is a smooth function whose Fourier transform is equal to 1 on the ball $|z|<(22 / 10)$ and vanishes outside the double of this ball.

Summing over $j$ and taking $L^{p}(\omega)$ norms yield

$$
\begin{aligned}
& \left\|T_{\sigma_{2}}\left(f_{1}, \ldots, f_{m-1}, f_{m}\right)\right\|_{L^{p}(\omega)} \\
& \leq C K \| \prod_{i=1}^{m-2}\left(M\left(M\left(f_{i}\right)^{\rho}\right)\right)^{1 / \rho} \sum_{j \in \mathbb{Z}}\left(M\left(\left|\Delta_{j}^{\prime}\left(f_{m-1}\right)\right|^{\rho}\right)\right)^{1 / \rho} \\
& \quad \times\left(M\left(\left|\Delta_{j}\left(f_{m}\right)\right|^{\rho}\right)\right)^{1 / \rho} \|_{L^{p}(\omega)} \\
& \leq C K \| \prod_{i=1}^{m-2}\left(M\left(M\left(f_{i}\right)^{\rho}\right)\right)^{1 / \rho} \\
& \quad \times\left\{\prod_{i=m-1}^{m} \sum_{j \in \mathbb{Z}}\left|M\left(\left|\Delta_{j}\left(f_{i}\right)\right|^{\rho}\right)\right|^{2 / \rho}\right\}^{1 / 2} \|
\end{aligned}
$$

where the last step holds due to the Cauchy-Schwarz inequality and we omitted the prime from the term with $i=m-1$ for the matter of simplicity. Applying Hölder's inequality and using that $\rho<2$ and Lemma 4 we obtain the conclusion that the expression above is bounded by

$$
C^{\prime} K\left\|f_{1}\right\|_{L^{p_{1}}(\omega)} \cdots\left\|f_{m}\right\|_{L^{p^{m}}(\omega)} .
$$

We next prove Theorem 1 under assumption (ii). It was proven in [6, page 136] that condition (6) is invariant under the adjoints; that is, it is also valid for the symbols of the dual operators $\sigma^{* m}\left(\xi_{1}, \ldots, \xi_{m}\right)=\sigma\left(\xi_{1}, \ldots, \xi_{m-1},-\left(\xi_{1}+\cdots+\xi_{m}\right)\right)$. To prove the required assertion, by duality, it is enough to prove that $T_{\sigma_{1}^{* m}}$ and $T_{\sigma_{2}^{* m}}$ are bounded from $L^{p_{1}}(\omega) \times \cdots \times$ $L^{p_{m-1}}(\omega) \times L^{p^{\prime}}\left(\omega^{1-p^{\prime}}\right)$ to $L^{p_{m}^{\prime}}\left(\omega^{1-p_{m}^{\prime}}\right)$. We may assume that $p_{m}=\min \left\{p_{1}, \ldots, p_{m}\right\}$. Since $p_{m}<(m n / \gamma)^{\prime}$, we see $1 / p^{\prime}$, $1 / p_{k}<1 / p^{\prime}+1 / p_{2}+\cdots+1 / p_{m}=1 / p_{m}^{\prime}<\gamma /(m n)$. Hence, $m n / \gamma<\min \left\{r, p^{\prime}, p_{1}, \ldots, p_{m-1}\right\}$. Since $p<p_{m}$ and $\omega^{1-p^{\prime}} \in A_{p^{\prime} \gamma /(m n)} \subset A_{p^{\prime}}$, we deduce that $\omega \in A_{p} \subset$ $A_{p_{m}}$; then $\omega^{1-p_{m}^{\prime}} \in A_{p_{m}^{\prime}}$. It is obvious that $\omega^{\left(1-p_{m}^{\prime}\right) / p_{m}^{\prime}}=$ $\omega^{-1 / p} \omega^{1 / p_{1}} \cdots \omega^{1 / p_{m}}$. Since $p_{m}<p_{k}, 1 / p=1 / p_{1}+\cdots+1 / p_{k}+$ 
$\cdots+1 / p_{m-1}>1 / p_{m}+1 / p_{k} \geq 2 / p_{k}$. That is, $p<p_{k} / 2$; then $\omega \in A_{p} \subset A_{p_{k} / 2} \subset A_{p_{k} \gamma /(m n)}$. Therefore, we take a positive number $\rho$ such that $1 \leq m n / \gamma<\rho<\min \left\{r, p^{\prime}, p_{1}\right.$, $\left.\ldots, p_{m-1}\right\}$, and $\gamma>m n / \rho$ such that $\omega^{1-p^{\prime}} \in A_{p^{\prime} / \rho}$ and $\omega \in A_{p_{k} / \rho}$. We have

$$
\begin{aligned}
& \left\|T_{\sigma_{1}^{* m}}\left(f_{1}, \ldots, f_{m-1}, f_{m}\right)\right\|_{L^{p^{\prime}\left(\omega^{1-p_{m}^{\prime}}\right)}} \\
& \leq C K \|\left\{\sum_{j} \mid T_{\sigma_{1}}\left(S_{j}\left(f_{1}\right), \ldots, S_{j}\left(f_{m-1}\right),\right.\right. \\
& \left.\left.\Delta_{j}\left(f_{m}\right)\right)\left.\right|^{2}\right\}^{1 / 2} \|_{L_{m}^{\prime}\left(\omega^{1-p_{m}^{\prime}}\right)} \\
& \leq C^{\prime} K\left\|\left\{\sum_{j} M\left(\left|\Delta_{j}\left(f_{m}\right)\right|^{\rho}\right)^{2 / \rho}\right\}^{1 / 2}\right\|_{L^{p^{\prime}}\left(\omega^{1-p^{\prime}}\right)} \\
& \times \prod_{i=1}^{m-1}\left\|\left(M\left(M\left(f_{i}\right)^{\rho}\right)\right)^{1 / \rho}\right\|_{L^{p_{i}}(\omega)} \\
& \leq C^{\prime \prime} K\left\|\left\{\sum_{j} M\left(\left|\Delta_{j}\left(f_{m}\right)\right|^{\rho}\right)^{2 / \rho}\right\}^{\rho / 2}\right\|_{L^{p^{\prime} / \rho}\left(\omega^{1-p^{\prime}}\right)}^{1 / \rho} \\
& \times \prod_{i=1}^{m-1}\left\|f_{i}\right\|_{L^{p_{i}}(\omega)} \\
& \leq C^{\prime} K\left\|f_{1}\right\|_{L^{p_{1}}(\omega)} \cdots\left\|f_{m}\right\|_{L^{p_{m}}(\omega)} .
\end{aligned}
$$

Similarly, we have

$$
\begin{aligned}
& \left\|T_{\sigma_{2}^{* m}}\left(f_{1}, \ldots, f_{m-1}, f_{m}\right)\right\|_{L_{p_{m}^{\prime}\left(\omega^{1-p_{m}^{\prime}}\right)}} \\
& \leq C K \| \prod_{i=1}^{m-2}\left(M\left(M\left(f_{i}\right)^{\rho}\right)\right)^{1 / \rho} \omega^{1 / p_{i}} \\
& \times \sum_{j \in \mathbb{Z}}\left(M\left(\left|\Delta_{j}^{\prime}\left(f_{m-1}\right)\right|^{\rho}\right)\right)^{1 / \rho} \\
& \times\left(M\left(\left|\Delta_{j}\left(f_{m}\right)\right|^{\rho}\right)\right)^{1 / \rho} \omega^{-1 / p+1 / p_{m-1}} \|_{L_{L_{m}^{\prime}\left(\omega^{1-p_{m}^{\prime}}\right)}} \\
& \leq C K\left\|\prod_{i=1}^{m-2}\left(M\left(M\left(f_{i}\right)^{\rho}\right)\right)^{1 / \rho}\right\|_{L^{p_{i}}\left(\omega_{i}\right)} \\
& \times\left\|\left\{\sum_{j \in \mathbb{Z}}\left|M\left(\left|\Delta_{j}\left(f_{m-1}\right)\right|^{\rho}\right)\right|^{2 / \rho}\right\}^{\rho / 2}\right\|_{L^{p_{m-1} / \rho}(\omega)}
\end{aligned}
$$

$$
\begin{aligned}
& \times\left\|\left\{\sum_{j \in \mathbb{Z}}\left|M\left(\left|\Delta_{j}\left(f_{m}\right)\right|^{\rho}\right)\right|^{2 / \rho}\right\}^{\rho / 2}\right\|_{L^{p^{\prime} / \rho\left(\omega^{1-p^{\prime}}\right)}} \\
\leq & C^{\prime} K\left\|f_{1}\right\|_{L^{p_{1}}(\omega)} \cdots\left\|f_{m}\right\|_{L^{p_{m}}(\omega)} .
\end{aligned}
$$

This concludes the proof of Theorem 1 .

\section{The Proof of Theorem 2}

We begin with some lemmas which will be used in the proof of Theorem 2 .

Lemma 6 (see [11]). Let $0<p$ and $\delta<\infty$ and let $\omega$ be a weight in $A_{\infty}$. Then, there exists $C>0$ (depending on the $A_{\infty}$ constant of $\omega$ ) such that

$$
\int_{R^{n}}\left(M_{\delta} f(x)\right)^{p} \omega(x) d x \leq C \int_{R^{n}}\left(M_{\delta}^{\sharp} f(x)\right)^{p} \omega(x) d x,
$$

for all function $f$ for which the left-hand side is finite.

Lemma 7 (see [13]). Let $0<p_{1}, p_{2}, p \leq \infty$, and $1 / p_{1}+1 / p_{2}=1 / p$. Let $\sigma$ be a multiplier satisfying $\sup _{k \in \mathbb{Z}}\left\|\sigma\left(2^{k} \cdot\right) \psi\right\|_{W^{s_{1}, s_{2}}\left(\mathbb{R}^{m n}\right)}<\infty$ for $s_{1}>\max \left\{n / 2, n / p_{1}-n / 2\right\}$, $s_{2}>\max \left\{n / 2, n / p_{2}-n / 2\right\}$, and $s_{1}+s_{2}>n / p_{1}+n / p_{2}-n / 2$; then $T_{\sigma}$ is bounded from $H^{p_{1}}\left(\mathbb{R}^{n}\right) \times H^{p_{2}}\left(\mathbb{R}^{n}\right)$ to $L^{p}\left(\mathbb{R}^{n}\right)$.

Remark 8. It should be pointed out that Lemma 7 can be extended to the case $m \geq 3$.

Lemma 9 (see [8]). Let $r>0, q_{1}, \ldots, q_{m} \in[2, \infty$ ), and $s_{1}, \ldots, s_{m} \geq 0$. Then there exists a constant $C>0$ such that

$$
\begin{aligned}
& \left(\int _ { \mathbb { R } ^ { n } } \ldots \left(\left(\int_{\mathbb{R}^{n}}\left|\widehat{F}\left(\xi_{1}, \ldots, \xi_{m}\right)\right|^{q_{1}}\left\langle\xi_{1}\right\rangle^{s_{1}} d \xi_{1}\right)^{q_{2} / q_{1}}\right.\right. \\
& \left.\left.\times\left\langle\xi_{2}\right\rangle^{s_{2}} d \xi_{2}\right)^{q_{3} / q_{2}} \cdots\left\langle\xi_{m}\right\rangle^{s_{m}} d \xi_{m}\right)^{1 / q_{m}} \\
& \leq C\|F\|_{W^{s_{1}} / q_{1}, \ldots, s_{m} / q_{m}\left(\mathbb{R}^{m n}\right)}
\end{aligned}
$$

for all $F \in W^{s_{1} / q_{1}, \ldots, s_{m}} / q_{m}\left(\mathbb{R}^{m n}\right)$ with $\operatorname{supp} F \quad \subset$ $\left\{\sqrt{\left|x_{1}\right|^{2}+\cdots+\left|x_{m}\right|^{2}} \leq r\right\}$.

Next, we give a pointwise control of $M_{\delta}^{\sharp} T_{\sigma}(\vec{f})$ which becomes very useful in the proof of Theorem 2 .

Lemma 10. Let $1<p_{1}, \ldots, p_{m}<2$. Assume that $\sigma \in$ $L^{\infty}\left(\mathbb{R}^{m n}\right)$ which satisfies $\sup _{k \in \mathbb{Z}}\left\|\sigma\left(2^{k} \cdot\right) \psi\right\|<\infty_{W^{s_{1}, \ldots, s_{m}}\left(\mathbb{R}^{m n}\right)}$ for $s_{1}>n / p_{1}, \ldots, s_{m}>n / p_{m}$ and $s_{1}+\cdots+s_{m}<n / p_{1}+$ $\cdots+n / p_{m}+1$. For any $0<\delta<1 / m$, one has $M_{\delta}^{\sharp} T_{\sigma}(\vec{f})(x) \leq$ $C \prod_{j=1}^{m} M_{p_{j}} f_{j}(x)$.

Proof. For simplicity, we only prove for the case $m=2$, since there is no essential difference for the general case. Fix an $x \in \mathbb{R}^{n}$ and a cube $Q$ with side length $l$, such that $x \in Q$. 
Let $f_{i}=f_{i}^{0}+f_{i}^{\infty}$, where $f_{i}^{0}=f_{i} \chi_{\mathrm{Q}^{*}}$ and $f_{i}^{\infty}=f_{i} \chi_{\left(\mathrm{Q}^{*}\right)^{c}}$ for $i=1,2$ and $Q^{*}=4 \sqrt{n} \mathrm{Q}$. Since $0<\delta<1 / 2$, we have

$$
\begin{aligned}
& \left(\left.\frac{1}{|Q|} \int_{Q}|| T_{\sigma}\left(f_{1}, f_{2}\right)(z)\right|^{\delta}-|C|^{\delta} \mid d z\right)^{1 / \delta} \\
& \leq C\left(\frac{1}{|Q|} \int_{Q}\left|T_{\sigma}\left(f_{1}, f_{2}\right)(z)-C\right|^{\delta} d z\right)^{1 / \delta} \\
& \leq C\left(\frac{1}{|Q|} \int_{Q} \mid T_{\sigma}\left(f_{1}^{0}, f_{2}^{0}\right)(z)\right. \\
& +T_{\sigma}\left(f_{1}^{\infty}, f_{2}^{\infty}\right)(z)+T_{\sigma}\left(f_{1}^{0}, f_{2}^{\infty}\right)(z) \\
& \left.+T_{\sigma}\left(f_{1}^{0}, f_{2}^{0}\right)(z)-\left.C\right|^{\delta} d z\right)^{1 / \delta} \\
& \leq C\left(\frac{1}{|Q|} \int_{Q}\left|T_{\sigma}\left(f_{1}^{0}, f_{2}^{0}\right)(z)\right|^{\delta} d z\right)^{1 / \delta} \\
& +\left(\frac{1}{|Q|} \int_{Q} \mid T_{\sigma}\left(f_{1}^{\infty}, f_{2}^{\infty}\right)(z)+T_{\sigma}\left(f_{1}^{0}, f_{2}^{\infty}\right)(z)\right. \\
& \left.+T_{\sigma}\left(f_{1}^{\infty}, f_{2}^{0}\right)(z)-\left.C\right|^{\delta} d z\right)^{1 / \delta} \\
& =U_{1}+U_{2} . \quad
\end{aligned}
$$

We first consider $U_{1}$. By Kolmogorov's inequality, Hölder's inequality, and Lemma 7, we have

$$
\begin{aligned}
& \left(\frac{1}{|\mathrm{Q}|} \int_{\mathrm{Q}}\left|T_{\sigma}\left(f_{1}^{0}, f_{2}^{0}\right)(z)\right|^{\delta} d z\right)^{1 / \delta} \\
& \quad \leq C\left\|T_{\sigma}\left(f_{1}^{0}, f_{2}^{0}\right)\right\|_{L^{p, \infty}(\mathrm{Q}, d x / \mathrm{QQ} \mid)}
\end{aligned}
$$

$$
\begin{aligned}
& \leq C \prod_{j=1}^{2}\left(\frac{1}{\left|Q^{*}\right|} \int_{Q^{*}}\left|f_{j}(z)\right|^{p_{j}} d z\right)^{1 / p_{j}} \\
& \leq C \prod_{j=1}^{2} M_{p_{j}} f_{j}(x)
\end{aligned}
$$

where $1 / p=1 / p_{1}+1 / p_{2}$ with $p>\delta$ and $1<p_{1}, p_{2}<\infty$.

Next we deal with $U_{2}$. We choose $C=\sum_{i=1}^{3} C_{i}$, where

$$
\begin{aligned}
& C_{1}=T_{\sigma}\left(f_{1}^{\infty}, f_{2}^{\infty}\right)(x), \\
& C_{2}=T_{\sigma}\left(f_{1}^{0}, f_{2}^{\infty}\right)(x), \\
& C_{3}=T_{\sigma}\left(f_{1}^{\infty}, f_{2}^{0}\right)(x) .
\end{aligned}
$$

We may split $U_{2}$ as $U_{2} \leq U_{21}+U_{22}+U_{23}$, where

$$
\begin{aligned}
& U_{21}=\left(\frac{1}{|Q|} \int_{Q}\left|T_{\sigma}\left(f_{1}^{\infty}, f_{2}^{\infty}\right)(z)-T_{\sigma}\left(f_{1}^{\infty}, f_{2}^{\infty}\right)(x)\right|^{\delta} d z\right)^{1 / \delta}, \\
& U_{22}=\left(\frac{1}{|Q|} \int_{Q}\left|T_{\sigma}\left(f_{1}^{0}, f_{2}^{\infty}\right)(z)-T_{\sigma}\left(f_{1}^{0}, f_{2}^{\infty}\right)(x)\right|^{\delta} d z\right)^{1 / \delta}, \\
& U_{23}=\left(\frac{1}{|Q|} \int_{Q}\left|T_{\sigma}\left(f_{1}^{\infty}, f_{2}^{0}\right)(z)-T_{\sigma}\left(f_{1}^{\infty}, f_{2}^{0}\right)(x)\right|^{\delta} d z\right)^{1 / \delta} .
\end{aligned}
$$

Now we estimate $U_{21}$ first. We decompose $\sigma$ as

$$
\sigma=\sum_{j \in \mathbb{Z}} \sigma(\cdot) \psi\left(\cdot / 2^{j}\right) \doteq \sum_{j \in \mathbb{Z}} \sigma_{j} .
$$

Let $\sigma_{j}=\sigma(\cdot) \psi\left(\cdot / 2^{j}\right)$, where $\psi \in \mathcal{S}\left(\mathbb{R}^{2 n}\right)$ with supp $\psi \subset$ $\left\{\xi \in \mathbb{R}^{2 n}: 1 / 2 \leq|\xi| \leq 2\right\}$ and $\sum_{j \in \mathbb{Z}} \psi\left(2^{-j} \xi\right)=1, \xi \neq 0$. Thus, we have

$$
\begin{aligned}
& \left|T_{\sigma}\left(f_{1}^{\infty}, f_{2}^{\infty}\right)(z)-T_{\sigma}\left(f_{1}^{\infty}, f_{2}^{\infty}\right)(x)\right| \\
& \quad \leq C \sum_{j \in \mathbb{Z}}\left|T_{\sigma_{j}}\left(f_{1}^{\infty}, f_{2}^{\infty}\right)(z)-T_{\sigma_{j}}\left(f_{1}^{\infty}, f_{2}^{\infty}\right)(x)\right| \\
& \quad \leq C \sum_{j \in \mathbb{Z}} \sum_{k_{1}=0}^{\infty} \sum_{k_{2}=0}^{\infty} \int_{2^{k_{2}+1} Q^{*} \mid 2^{k_{2}} Q^{*}} \int_{2^{k_{1}+1} Q^{*} \mid 2^{k_{1}} Q^{*}}\left|\sigma_{j}^{\vee}\left(z-y_{1}, z-y_{2}\right)-\sigma_{j}^{\vee}\left(x-y_{1}, x-y_{2}\right)\right|\left|f_{1}\left(y_{1}\right) f_{2}\left(y_{2}\right)\right| d y_{1} d y_{2} \\
& \quad \leq C \sum_{k_{1}=0}^{\infty} \sum_{k_{2}=0}^{\infty} \sum_{j \in \mathbb{Z}} \int_{2^{k_{2}+1} Q^{*} \mid 2^{k_{2}} Q^{*}} \int_{2^{k_{1}+1} Q^{*} \mid 2^{k_{1}} Q^{*}}\left|\sigma_{j}^{\vee}\left(z-y_{1}, z-y_{2}\right)-\sigma_{j}^{\vee}\left(x-y_{1}, x-y_{2}\right)\right|\left|f_{1}\left(y_{1}\right) f_{2}\left(y_{2}\right)\right| d y_{1} d y_{2} \\
& \quad \leq \sum_{k_{1}=0}^{\infty} \sum_{k_{2}=0}^{\infty} I_{k_{1}, k_{2}} .
\end{aligned}
$$


Abstract and Applied Analysis

7

Applying Hölder's inequality we have

$$
\begin{aligned}
I_{k_{1}, k_{2}}= & \sum_{j \in \mathbb{Z}} \int_{2^{k_{2}+1} Q^{*} \mid 2^{k_{2} Q^{*}}} \int_{2^{k_{1}+1} Q^{*} \mid 2^{k_{1} Q^{*}}}\left|\sigma_{j}^{\vee}\left(z-y_{1}, z-y_{2}\right)-\sigma_{j}^{\vee}\left(x-y_{1}, x-y_{2}\right)\right|\left|f_{1}\left(y_{1}\right) f_{2}\left(y_{2}\right)\right| d y_{1} d y_{2} \\
\leq & C \sum_{j \in \mathbb{Z}}\left(\int_{2^{k_{2}+1} Q^{*} \mid 2^{k_{2} Q^{*}}}\left(\int_{2^{k_{1}+1} Q^{*} \mid 2^{k_{1} Q^{*}}}\left|\sigma_{j}^{\vee}\left(z-y_{1}, z-y_{2}\right)-\sigma_{j}^{\vee}\left(x-y_{1}, x-y_{2}\right)\right|^{p_{1}^{\prime}} d y_{1}\right)^{p_{2}^{\prime} \mid p_{1}^{\prime}} d y_{2}\right)^{1 / p_{2}^{\prime}} \\
& \times\left(\int_{2^{k_{1}+1} Q^{*}}\left|f_{1}\left(y_{1}\right)\right|^{p_{1}} d y_{1}\right)^{1 / p_{1}}\left(\int_{2^{k_{2}+1} Q^{*}}\left|f_{2}\left(y_{2}\right)\right|^{p_{2}} d y_{2}\right)^{1 / p_{2}} \\
\doteq & C \sum_{j \in \mathbb{Z}} I_{k_{1}, k_{2}, j} \times\left(\int_{2^{k_{2}+1} Q^{*}}\left|f_{1}\left(y_{1}\right)\right|^{p_{1}} d y_{1}\right)^{1 / p_{1}}\left(\int_{2^{k_{2}+1} Q^{*}}\left|f_{2}\left(y_{2}\right)\right|^{p_{2}} d y_{2}\right)^{1 / p_{2}} .
\end{aligned}
$$

Let $h=z-x$ and $\widetilde{Q}=x-Q^{*}$. Then we have

$$
\begin{aligned}
& I_{k_{1}, k_{2}, j}=\left(\int_{2^{k_{2}+1} Q^{*} \backslash 2^{k_{2}} Q^{*}}\left(\int_{2^{k_{1}+1} Q^{*} \backslash 2^{k_{1}} Q^{*}}\left|\sigma_{j}^{\vee}\left(z-y_{1}, z-y_{2}\right)-\sigma_{j}^{\vee}\left(x-y_{1}, x-y_{2}\right)\right|^{p_{1}^{\prime}} d y_{1}\right)^{p_{2}^{\prime} / p_{1}^{\prime}} d y_{2}\right)^{1 / p_{2}^{\prime}} \\
& \leq C\left(\int_{2^{k_{2}+1} \widetilde{\mathrm{Q}} \mid 2^{k_{2}} \widetilde{\mathrm{Q}}}\left(\int_{2^{k_{1}+1} \widetilde{\mathrm{Q}} \mid 2^{k_{1}} \widetilde{\mathrm{Q}}}\left|\sigma_{j}^{\vee}\left(h+y_{1}, h+y_{2}\right)-\sigma_{j}^{\vee}\left(y_{1}, y_{2}\right)\right|^{p_{1}^{\prime}} d y_{1}\right)^{p_{2}^{\prime} / p_{1}^{\prime}} d y_{2}\right)^{1 / p_{2}^{\prime}} \\
& \leq C\left(\int_{2^{k_{2}+1} \widetilde{Q} \backslash 2^{k_{2}} \widetilde{Q}}\left(\int_{2^{k_{1}+1} \widetilde{Q} \backslash 2^{k_{1}} \widetilde{Q}}\left|\sigma_{j}^{\vee}\left(y_{1}, y_{2}\right)\right|^{p_{1}^{\prime}} d y_{1}\right)^{p_{2}^{\prime} / p_{1}^{\prime}} d y_{2}\right)^{1 / p_{2}^{\prime}} \\
& \leq C\left(2^{k_{1}} l\right)^{-s_{1}}\left(2^{k_{2}} l\right)^{-s_{2}}\left(\int _ { 2 ^ { k _ { 2 } + 1 } \widetilde { \mathrm { Q } } \backslash 2 ^ { k _ { 2 } } \widetilde { \mathrm { Q } } } \left(\int_{2^{k_{1}+1} Q^{*} \backslash 2^{k_{1}} \widetilde{\mathrm{Q}}}\left|\sigma_{j}^{\vee}\left(y_{1}, y_{2}\right)\right|^{p_{1}^{\prime}}\right.\right. \\
& \left.\left.\times\left(1+\left|y_{1}\right|^{2}\right)^{s_{1} p_{1}^{\prime} / 2} d y_{1}\right)^{p_{2}^{\prime} / p_{1}^{\prime}}\left(1+\left|y_{2}\right|^{2}\right)^{s_{2} p_{2}^{\prime} / 2} d y_{2}\right)^{1 / p_{2}^{\prime}} \\
& \leq C\left(2^{k_{1}} l\right)^{-s_{1}}\left(2^{k_{2}} l\right)^{-s_{2}}\left(\int _ { \mathbb { R } ^ { n } } \left(\int_{\mathbb{R}^{n}}\left|\sigma_{j}^{\vee}\left(2^{-j} y_{1}, 2^{-j} y_{2}\right)\right|^{p_{1}^{\prime}}\right.\right. \\
& \left.\left.\times\left(1+\left|2^{-j} y_{1}\right|^{2}\right)^{s_{1} p_{1}^{\prime} / 2} 2^{-j n} d y_{1} \times y_{1}\right)^{p_{2}^{\prime} / p_{1}^{\prime}}\left(1+\left|2^{-j} y_{2}\right|^{2}\right)^{s_{2} p_{2}^{\prime} / 2} 2^{-j n} d y_{2}\right)^{1 / p_{2}^{\prime}}
\end{aligned}
$$




$$
\begin{aligned}
& \leq C\left(2^{k_{1}} l\right)^{-s_{1}}\left(2^{k_{2}} l\right)^{-s_{2}} 2^{-j\left(s_{1}+s_{2}\right)} 2^{j n\left(\left(1 / p_{1}\right)+\left(1 / p_{2}\right)\right)} \\
& \quad \times\left(\int_{\mathbb{R}^{n}}\left(\int_{\mathbb{R}^{n}}\left|2^{-2 j n} \sigma_{j}^{\vee}\left(2^{-j} y_{1}, 2^{-j} y_{2}\right)\right|^{p_{1}^{\prime}}\left(1+\left|y_{1}\right|^{2}\right)^{s_{1} p_{1}^{\prime} / 2} d y_{1}\right)^{p_{2}^{\prime} / p_{1}^{\prime}}\left(1+\left|y_{2}\right|^{2}\right)^{s_{2} p_{2}^{\prime} / 2} d y_{2}\right)^{1 / p_{2}^{\prime}} \\
& \leq C\left(2^{k_{1}} l\right)^{-s_{1}}\left(2^{k_{2}} l\right)^{-s_{2}} 2^{-j\left(s_{1}+s_{2}\right)} 2^{j n\left(\left(1 / p_{1}\right)+\left(1 / p_{2}\right)\right)}\left\|\sigma\left(2^{j} \cdot\right) \psi\right\|_{W^{s_{1}, s_{2}}},
\end{aligned}
$$

where the last inequality holds due to Lemma 9. Suppose that $2^{-R} \leq l<2^{-R+1}$. Since $n / p_{1}+n / p_{2}-s_{1}-s_{2}<0$, we have

$$
\sum_{j \geq R} I_{k_{1}, k_{2}, j} \leq \sup _{j}\left\|\sigma\left(2^{j} \cdot\right) \psi\right\|_{W^{s_{1}, s_{2}}}
$$

$$
\begin{array}{r}
\times \sum_{j \geq R}\left(2^{k_{1}} l\right)^{-s_{1}}\left(2^{k_{2}} l\right)^{-s_{2}} 2^{j\left(n / p_{1}+n / p_{2}-s_{1}-s_{2}\right)} \\
\leq C \sup _{j}\left\|\sigma\left(2^{j} \cdot\right) \psi\right\|_{W^{s_{1}, s_{2}}} 2^{-k_{1} s_{1}} 2^{-k_{2} s_{2}} l^{-n / p_{1}} l^{-n / p_{2}} .
\end{array}
$$

On the other hand

$$
\begin{aligned}
& I_{k_{1}, k_{2}, j} \leq\left(\int_{2^{k_{2}+1} \widetilde{\mathrm{Q}} \backslash 2^{k_{2}} \widetilde{\mathrm{Q}}}\left(\int_{2^{k_{1}+1} \widetilde{\mathrm{Q}} \backslash 2^{k_{1}} \widetilde{\mathrm{Q}}}\left|\sigma_{j}^{\vee}\left(y_{1}+h, y_{2}+h\right)-\sigma_{j}^{\vee}\left(y_{1}, y_{2}\right)\right|^{p_{1}^{\prime}} d y_{1}\right)^{p_{2}^{\prime} / p_{1}^{\prime}} d y_{2}\right)^{1 / p_{2}^{\prime}} \\
& \leq C\left(\int_{2^{k_{2}+1} \widetilde{\mathrm{Q} \backslash 2^{k_{2}} \widetilde{\mathrm{Q}}}}\left(\int_{2^{k_{1}+1} \widetilde{\mathrm{Q} \backslash 2^{k_{1}} \widetilde{\mathrm{Q}}}}\left(\int_{0}^{1}\left|\vec{h} \cdot \nabla\left(\sigma_{j}^{\vee}\right)\left(y_{1}+\theta h, y_{2}+\theta h\right)\right| d \theta\right)^{p_{1}^{\prime}} d y_{1}\right)^{p_{2}^{\prime} / p_{1}^{\prime}} d y_{2}\right)^{1 / p_{2}^{\prime}} \\
& \leq C \int_{0}^{1}\left(\int_{2^{k_{2}+1} \widetilde{\mathrm{Q}} \backslash 2^{k_{2}} \widetilde{\mathrm{Q}}}\left(\int_{2^{k_{1}+1} \widetilde{\mathrm{Q}} \backslash 2^{k_{1}} \widetilde{\mathrm{Q}}}\left|\vec{h} \cdot \nabla\left(\sigma_{j}^{\vee}\right)\left(y_{1}+\theta h, y_{2}+\theta h\right)\right|^{p_{1}^{\prime}} d y_{1}\right)^{p_{2}^{\prime} / p_{1}^{\prime}} d y_{2}\right)^{1 / p_{2}^{\prime}} d \theta \\
& \leq C\left(\int_{2^{k_{2}+1} \widetilde{\mathrm{Q}} \backslash 2^{k_{2}} \widetilde{\mathrm{Q}}}\left(\int_{2^{k_{1}+1} \widetilde{\mathrm{Q}} \backslash 2^{k_{1}} \widetilde{\mathrm{Q}}}\left|\vec{h} \cdot \nabla\left(\sigma_{j}^{\vee}\right)\left(y_{1}, y_{2}\right)\right|^{p_{1}^{\prime}} d y_{1}\right)^{p_{2}^{\prime} / p_{1}^{\prime}} d y_{2}\right)^{1 / p_{2}^{\prime}}
\end{aligned}
$$

where $\vec{h}=(h, h) \in \mathbb{R}^{2 n}$. Since $\vec{h} \cdot \nabla\left(\sigma_{j}^{\vee}\right)\left(y_{1}, y_{2}\right)=$ $\sum_{r=1}^{2 n} h_{r} \partial_{r}\left(\sigma_{j}^{\vee}\right)\left(y_{1}, y_{2}\right)$, we have

$$
\begin{gathered}
I_{k_{1}, k_{2}, j} \leq C \sum_{r=1}^{2 n} l\left(\int_{2^{k_{2}+1} \widetilde{\mathrm{Q}} \backslash 2^{k_{2}} \widetilde{\mathrm{Q}}}\left(\int_{2^{k_{1}+1} \widetilde{\mathrm{Q}} \backslash 2^{k_{1}} \widetilde{\mathrm{Q}}}\left|\partial_{r}\left(\sigma_{j}^{\vee}\right)\left(y_{1}, y_{2}\right)\right|^{p_{1}^{\prime}} d y_{1}\right)^{p_{2}^{\prime} / p_{1}^{\prime}} d y_{2}\right)^{1 / p_{2}^{\prime}} \\
\leq C \sum_{r=1}^{2 n} l\left(2^{k_{1}} l\right)^{-s_{1}}\left(2^{k_{2}} l\right)^{-s_{2}}\left(\int_{\mathbb{R}^{n}}\left(\int_{\mathbb{R}^{n}}\left|\partial_{r}\left(\sigma_{j}^{\vee}\right)\left(2^{-j} y_{1}, 2^{-j} y_{2}\right)\right|^{p_{1}^{\prime}}\left(1+\left|2^{-j} y_{2}\right|^{2}\right)^{s_{1} p_{1}^{\prime} / 2} 2^{-j n} d y_{1}\right)^{p_{2}^{\prime} / p_{1}^{\prime}}\right. \\
\left.\times\left(1+\left|2^{-j} y_{2}\right|^{2}\right)^{s_{2} p_{2}^{\prime} / 2} 2^{-j n} d y_{2}\right)^{1 / p_{2}^{\prime}}
\end{gathered}
$$




$$
\begin{gathered}
\leq C \sum_{r=1}^{2 n} l\left(2^{k_{1}} l\right)^{-s_{1}}\left(2^{k_{2}} l\right)^{-s_{2}}\left(\int_{\mathbb{R}^{n}}\left(\int_{\mathbb{R}^{n}}\left|2^{-2 j n} \partial_{r}\left(\sigma_{j}^{\vee}\right)\left(2^{-j} y_{1}, 2^{-j} y_{2}\right)\right|^{p_{1}^{\prime}}\left(1+\left|y_{1}\right|^{2}\right)^{s_{1} p_{1}^{\prime} / 2} d y_{1}\right)^{p_{2}^{\prime} / p_{1}^{\prime}}\right. \\
\left.\times\left(1+\left|y_{2}\right|^{2}\right)^{s_{2} p_{2}^{\prime} / 2} d y_{2}\right)^{1 / p_{2}^{\prime}} 2^{-j\left(s_{1}+s_{2}\right)} 2^{j n\left(\left(1 / p_{1}\right)+\left(1 / p_{2}\right)\right)} \\
\leq C \sum_{r=1}^{2 n} l\left(2^{k_{1}} l\right)^{-s_{1}}\left(2^{k_{2}} l\right)^{-s_{2}} 2^{-j\left(s_{1}+s_{2}\right)} 2^{j n\left(\left(1 / p_{1}\right)+\left(1 / p_{2}\right)\right)} 2^{j}\left\|\sigma\left(2^{j} \cdot\right) \psi\right\|_{W^{s_{1}, s_{2}}},
\end{gathered}
$$

where in the last inequality Lemma 9 was used again and hence

$$
\begin{aligned}
& \sum_{j<R} I_{k_{1}, k_{2}, j} \\
& \quad \leq C \sup _{j}\left\|\sigma\left(2^{j} \cdot\right) \psi\right\|_{W^{s_{1}, s_{2}}} 2^{-k_{1} s_{1}} 2^{-k_{2} s_{2}} l^{-n p_{1}} l^{-n / p_{2}} .
\end{aligned}
$$

Combining the above arguments we have

$$
\begin{gathered}
\left|T_{\sigma}\left(f_{1}^{\infty}, f_{2}^{\infty}\right)(z)-T_{\sigma}\left(f_{1}^{\infty}, f_{2}^{\infty}\right)(x)\right| \\
\leq C \sum_{k_{1}=0}^{\infty} \sum_{k_{2}=0}^{\infty} 2^{-k_{1} s_{1}} 2^{-k_{2} s_{2}} l^{-n / p_{1}} l^{-n / p_{2}} \\
\quad \times\left(\int_{2^{k_{1}+1} Q^{*}}\left|f_{1}\left(y_{1}\right)\right|^{p_{1}} d y_{1}\right)^{1 / p_{1}}
\end{gathered}
$$

$$
\begin{aligned}
& \left|T_{\sigma}\left(f_{1}^{0}, f_{2}^{\infty}\right)(z)-T_{\sigma}\left(f_{1}^{0}, f_{2}^{\infty}\right)(x)\right| \\
& \leq C \sum_{j}\left|T_{\sigma_{j}}\left(f_{1}^{0}, f_{2}^{\infty}\right)(z)-T_{\sigma_{j}}\left(f_{1}^{0}, f_{2}^{\infty}\right)(x)\right| \\
& \leq C \sum_{j} \sum_{k_{2}=0}^{\infty} \int_{2^{k_{2}+1} Q^{*}}\left|f_{2}\left(y_{2}\right)\right| \int_{Q^{*}}\left|\sigma_{j}^{\vee}\left(z-y_{1}, z-y_{2}\right)-\sigma_{j}^{\vee}\left(x-y_{1}, x-y_{2}\right)\right|\left|f_{1}\left(y_{1}\right)\right| d y_{1} d y_{2} \\
& \leq C \sum_{j \in \mathbb{Z} k_{2}=0}^{\infty}\left(\int_{2^{k_{2}+1} Q^{*} \backslash 2^{k_{2}} Q^{*}}\left(\int_{Q^{*}}\left|\sigma_{j}^{\vee}\left(z-y_{1}, z-y_{2}\right)-\sigma_{j}^{\vee}\left(x-y_{1}, x-y_{2}\right)\right|^{p_{1}^{\prime}} d y_{1}\right)^{p_{2}^{\prime} / p_{1}^{\prime}} d y_{2}\right)^{1 / p_{2}^{\prime}} \\
& \quad \times\left(\int_{Q^{*}}\left|f_{1}\left(y_{1}\right)\right|^{p_{1}} d y_{1}\right)^{1 / p_{1}}\left(\int_{2^{k_{2}+1} Q^{*}}\left|f_{2}\left(y_{2}\right)\right|^{p_{2}} d y_{2}\right)^{1 / p_{2}} .
\end{aligned}
$$

$$
\begin{aligned}
& \times\left(\int_{2^{k_{2}+1} Q^{*}}\left|f_{2}\left(y_{2}\right)\right|^{p_{2}} d y_{2}\right)^{1 / p_{2}} \\
\leq & C \sum_{k_{1}=0}^{\infty} 2^{-k_{1}\left(s_{1}-n / p_{1}\right)} \\
& \times \sum_{k_{2}=0}^{\infty} 2^{-k_{2}\left(s_{2}-n / p_{2}\right)} M_{p_{1}} f_{1} M_{p_{2}} f_{2} \\
\leq & C M_{p_{1}} f_{1} M_{p_{2}} f_{2} .
\end{aligned}
$$

Thus, we obtain $U_{21} \leq C M_{p_{1}} f_{1} M_{p_{2}} f_{2}$. What remain to be considered are $U_{22}$ and $U_{23}$. We just estimate $U_{22}$ since the same arguments can be applied to $U_{23}$ :
Then by similar arguments as the above mentioned we get

that

$$
\begin{aligned}
& \left|T_{\sigma}\left(f_{1}^{0}, f_{2}^{\infty}\right)(z)-T_{\sigma}\left(f_{1}^{0}, f_{2}^{\infty}\right)(x)\right| \\
& \quad \leq C \sum_{k_{2}=0}^{\infty} 2^{-k_{2} s_{2}} l^{-n / p_{1}} l^{-n / p_{2}}\left(\int_{Q^{*}}\left|f_{1}\left(y_{1}\right)\right|^{p_{1}} d y_{1}\right)^{1 / p_{1}}
\end{aligned}
$$

$$
\begin{aligned}
& \times\left(\int_{2^{k_{2}+1} Q^{*}}\left|f_{2}\left(y_{2}\right)\right|^{p_{2}} d y_{2}\right)^{1 / p_{2}} \\
\leq & C \sum_{k_{2}=0}^{\infty} 2^{-k_{2}\left(s_{2}-p_{2}\right)} M_{p_{1}} f_{1} M_{p_{2}} f_{2} \\
\leq & C M_{p_{1}} f_{1} M_{p_{2}} f_{2} .
\end{aligned}
$$

The proof of Lemma 10 is complete. 
Now we are ready to give the proof of Theorem 2.

Proof. By Lemma 3, we can choose $1<p_{1}<q_{1}$ and $1<$ $p_{2}<q_{2}$ such that $\omega_{1}^{q_{1}} \in A_{q_{1} / p_{1}}$ and $\omega_{2}^{q_{2}} \in A_{q_{2} / p_{2}}$. Then by the Hölder inequality, Lemma 10, and the weighted boundedness of $M$, we deduce that

$$
\begin{aligned}
\left\|T_{\sigma}\left(f_{1}, f_{2}\right)\right\|_{L^{q}\left(w^{q}\right)} & \leq\left\|M_{\delta} T_{\sigma}\left(f_{1}, f_{2}\right)\right\|_{L^{q}\left(w^{q}\right)} \\
& \leq C\left\|M_{\delta}^{\sharp} T_{\sigma}\left(f_{1}, f_{2}\right)\right\|_{L^{q}\left(w^{q}\right)} \\
& \leq C\left\|M_{p_{1}} f_{1} M_{p_{2}} f_{2}\right\|_{L^{q}\left(w^{q}\right)} \\
& \leq C\left\|M_{p_{1}} f_{1}\right\|_{L^{q_{1}}\left(w_{1}^{q_{1}}\right)}\left\|M_{p_{1}} f_{1}\right\|_{L^{q_{2}}\left(w_{2}^{q_{2}}\right)} \\
& \leq C\left\|f_{1}\right\|_{L^{q_{1}}\left(w_{1}^{q_{1}}\right)}\left\|f_{2}\right\|_{L^{q_{2}}\left(w_{2}^{q_{2}}\right)} .
\end{aligned}
$$

The proof of Theorem 2 is complete.

\section{Conflict of Interests}

The author declares that there is no conflict of interests regarding the publication of this paper.

\section{Acknowledgments}

This work was supported by the National Nature Science Foundation of China (nos. 11226102 and 11226103) and Doctor Foundation of Henan Polytechnic University (no. B2012055).

\section{References}

[1] R. R. Coifman and Y. Meyer, "Au delà des opérateurs pseudodifférentiels," Astérisque, vol. 57, pp. 1-185, 1978.

[2] L. Grafakos and R. H. Torres, "Multilinear Calderón-Zygmund theory," Advances in Mathematics, vol. 165, no. 1, pp. 124-164, 2002.

[3] C. E. Kenig and E. M. Stein, "Multilinear estimates and fractional integration," Mathematical Research Letters, vol. 6, no. 1, pp. 1-15, 1999.

[4] J. Duoandikoetxea, Fourier Analysis, vol. 29 of Graduate Studies in Mathematics, American Mathematical Society, Providence, RI, USA, 2001.

[5] N. Tomita, "A Hörmander type multiplier theorem for multilinear operators," Journal of Functional Analysis, vol. 259, no. 8, pp. 2028-2044, 2010.

[6] L. Grafakos and Z. Si, "The Hörmander multiplier theorem for multilinear operators," Journal für die Reine und Angewandte Mathematik, vol. 668, pp. 133-147, 2012.

[7] L. Grafakos, A. Miyachi, and N. Tomita, "On multilinear Fourier multipliers of limited smoothness," Canadian Journal of Mathematics, vol. 65, no. 2, pp. 299-330, 2013.

[8] M. Fujita and N. Tomita, "Weighted norm inequalities for multilinear Fourier multipliers," Transactions of the American Mathematical Society, vol. 364, no. 12, pp. 6335-6353, 2012.

[9] G. Hu and C. C. Lin, "Weighted norm inequalities for multilinear singular integral operators and applications," Analysis and Applications. In press, http://arxiv.org/abs/1208.6346.
[10] W. Li, Q. Xue, and K. Yabuta, "Weighted version of Carleson measure and multilinear Fourier multiplier," Forum Mathematicum, 2012.

[11] E. M. Stein, Harmonic Analysis: Real-Variable Methods, Orthogonality, and Oscillatory Integrals, vol. 43 of Princeton Mathematical Series, Princeton University Press, Princeton, NJ, USA, 1993.

[12] K. F. Andersen and R. T. John, "Weighted inequalities for vector-valued maximal functions and singular integrals," Studia Mathematica, vol. 69, no. 1, pp. 19-31, 1980/81.

[13] A. Miyachi and N. Tomita, "Minimal smoothness conditions for bilinear Fourier multipliers," Revista Matemática Iberoamericana, vol. 29, no. 2, pp. 495-530, 2013. 


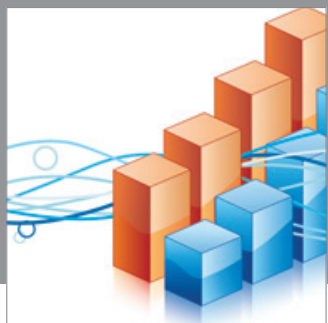

Advances in

Operations Research

mansans



The Scientific World Journal
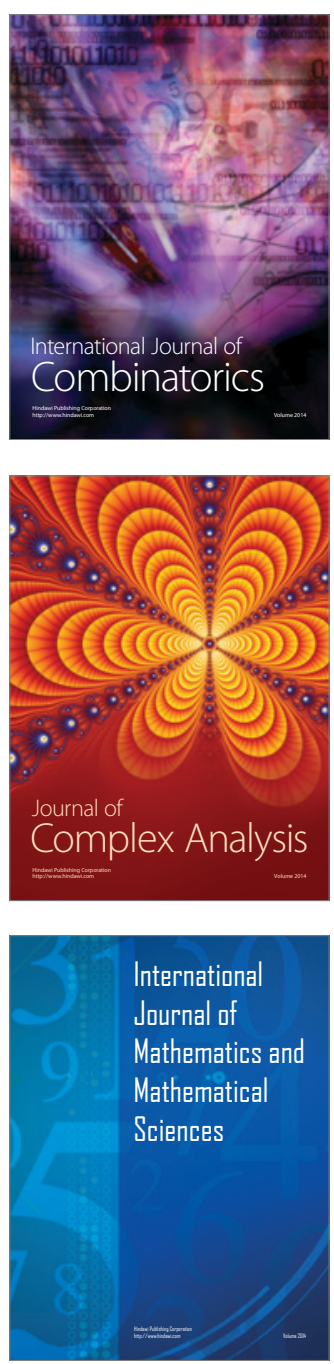
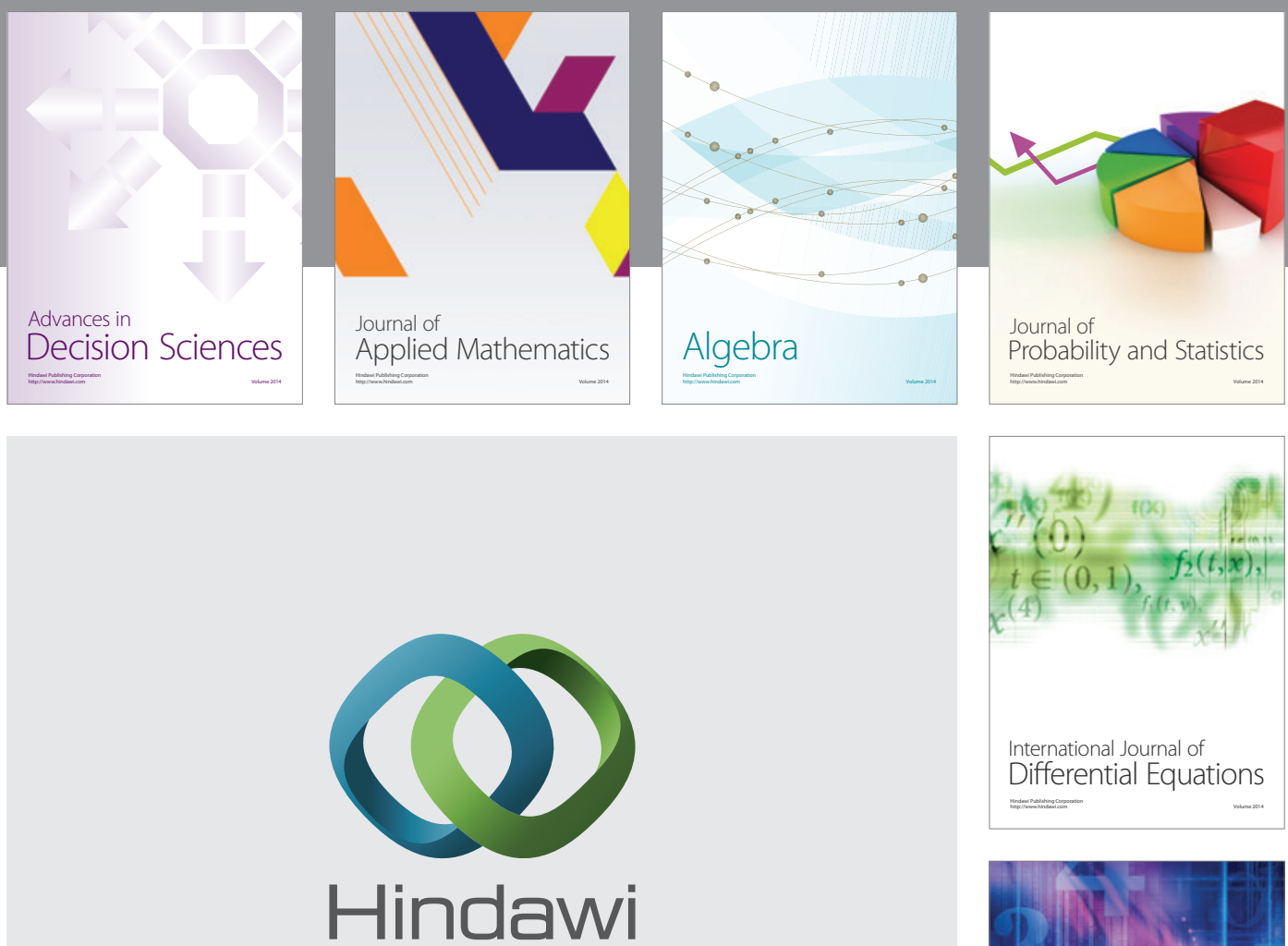

Submit your manuscripts at http://www.hindawi.com
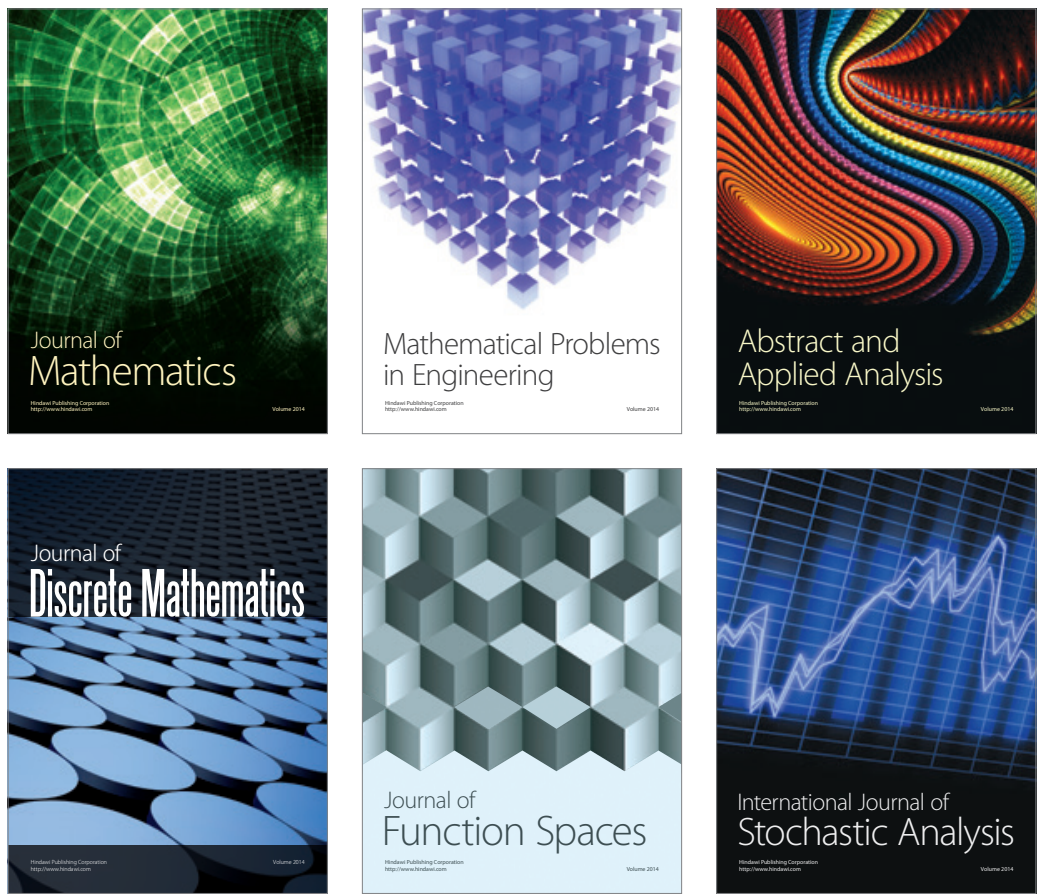

Journal of

Function Spaces

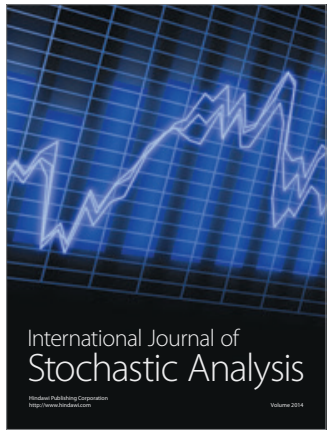

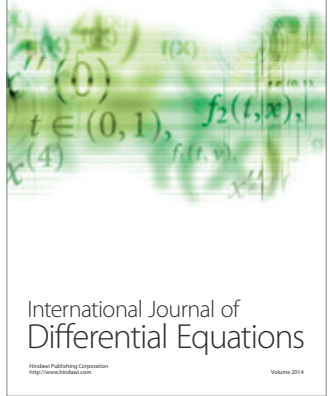
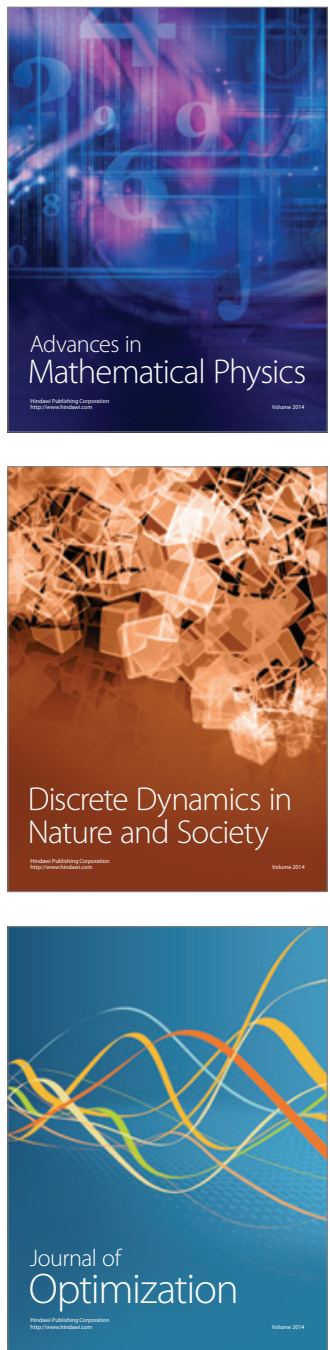